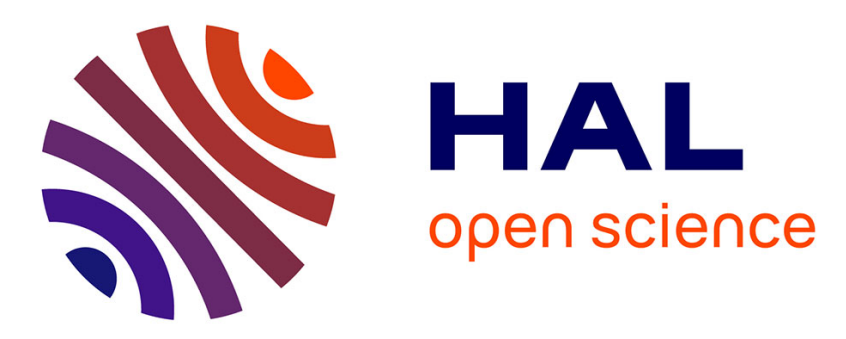

\title{
EMG-to-force estimation with full-scale physiology based muscle model
}

\author{
Mitsuhiro Hayashibe, David Guiraud, Philippe Poignet
}

\section{To cite this version:}

Mitsuhiro Hayashibe, David Guiraud, Philippe Poignet. EMG-to-force estimation with full-scale physiology based muscle model. IROS'09: International Conference on Intelligent RObots and Systems, Oct 2009, St. Louis, MO, United States. pp.1621-1626, 10.1109/IROS.2009.5354644 . lirmm00429594

\section{HAL Id: lirmm-00429594 https://hal-lirmm.ccsd.cnrs.fr/lirmm-00429594}

Submitted on 3 Nov 2009

HAL is a multi-disciplinary open access archive for the deposit and dissemination of scientific research documents, whether they are published or not. The documents may come from teaching and research institutions in France or abroad, or from public or private research centers.
L'archive ouverte pluridisciplinaire HAL, est destinée au dépôt et à la diffusion de documents scientifiques de niveau recherche, publiés ou non, émanant des établissements d'enseignement et de recherche français ou étrangers, des laboratoires publics ou privés. 


\title{
EMG-to-force estimation with full-scale physiology based muscle model
}

\author{
Mitsuhiro Hayashibe, David Guiraud, Philippe Poignet
}

\begin{abstract}
EMG-to-force estimation for voluntary muscle contraction has many applications in human-machine interaction, motion analysis, and rehabilitation robotics for prosthetic limbs or exoskeletons. EMG-based model can account for a subject's individual activation patterns to estimate muscle force. For the estimation, so-called Hill-type model has been used in most of the cases. It already has shown its promising performance, but it is still known as a phenomenological model considering only macroscopic physiology. We have already developed the physiological based muscle model for the use of functional electrical stimulation (FES) which can render the myoelectrical property also in microscopic scale. In this paper we discuss EMG-to-force estimation based on this full physiological based muscle model in voluntary contraction. In addition to Hill macroscopic structure, a microscopic physiology originally designed by Huxley is integrated. It has significant meaning to realize the same kind of EMG-toforce estimation with a physiological based model not with a phenomenological model, because it brings the understanding of the internal biophysical dynamics and new insights about neuromuscular activations. Using same EMG data of isometric muscle contraction, the force estimation results are shown by classical approach and new physiological based approach. Its interpretation is also discussed.
\end{abstract}

\section{INTRODUCTION}

Results of complex information processing in the human brain can be observed as the human motions originated by skeletal muscle contractions. Central nervous system has a fundamental role in the control of skeletal muscle together with peripheral nervous system. For the detailed understanding of brain mechanism, there is still a very long way to be taken for the future. At least for the preparation, neuromusculoskeletal system should be analyzed and modeled from microscopic scale to macroscopic scale as the pathway from neural activity to muscle activity. Thus, neuromusculoskeletal modeling is important for brain science to understand how limb movements are controlled smoothly and effectively[1][2]. It has also significant meanings for the clinical research of tremor [3] concerning spasticity induced by stroke or cerebral palsy. In addition, quantitative analysis between neural activity and muscle force can contribute to the design of Functional Electrical Stimulation (FES) for paralyzed muscles and myoelectrical limb control [4][5], and also to the design of robotic prosthetic limbs and exoskeletons. The general musculoskeletal model of whole body and its dynamics computation method were established and available [6]. The efficient computational algorithms were pursued for inverse and forward dynamics on the basis of efficient multibody dynamics computations [7].

The authors are with INRIA Sophia-Antipolis -DEMAR Project and LIRMM, UMR5506 CNRS UM2, 161 Rue Ada - 34392 Montpellier Cedex 5, France hayashibe, guiraud, poignet@lirmm. fr
In this paper, we focus on the physiological details of activated muscle unit. Especially we aim at developing EMGbased muscle model to study voluntary muscle contraction. EMG-based model relies on measured muscle activity to estimate muscle force. This method can account for a subject's individual activation patterns and its characteristics. EMGbased models have been used to estimate torques around the joints [8]. Most of their muscle model have been based on phenomenological models derived from A. V. Hill's classic work [9] and well summarized by Zajac [10]. This muscle model has been used for a long time and by many groups. It can represent the muscle force accounting for the muscle activation level. It was modified and improved to be used for EMG-based muscle model. Many studies have shown the promise of EMG-driven musculoskeletal models to predict human joint moments [11][12]. Hill-Zajac macroscopic model is the standard promising muscle model for practical use. For the more physiologically and biophysically detailed model, a microscopic model was designed by Huxley in 1957 [13]. He proposed an explanation of the interaction crossbridge in a sarcomere. The distinctions between microscopic and macroscopic are not absolute; thus a sarcomere model can be used to represent a whole muscle which is assumed to be a homogeneous assembly of identical sarcomeres. Conversely, the Hill model which is developed originally for whole muscles have been used to represent the dynamics of individual sarcomeres within a fiber [14]. The distributionmoment model of Zahalak [15] is a model for sarcomeres or whole muscle which is extracted via a formal mathematical approximation from Huxley cross-bridge models. This model constitutes a bridge between the microscopic and macroscopic levels. Based on Huxley and Hill-type models, BestelSorine [16] proposed an explanation of how the beating of cardiac muscle may be performed, through a chemical control input, connected to the calcium dynamics in muscle cell, that stimulates the contractile element of the model. Starting with this concept, we first adapted it to the striated muscle under FES [17]. In this paper, we try to realize EMG-to-force estimation based on this physiological based muscle model in voluntary contraction. It is already known that EMG-toforce estimation by classical Hill approach is promising. It has significant meaning to realize the same kind of EMG-toforce estimation with a physiological based model not with a phenomenological model because it brings the understanding of the internal biophysical dynamics and new insights about neuromuscular activations. Using same data set of isometric muscle contraction, the force estimation results are shown by classical approach and new physiological based approach. 


\section{CLASSICAL MUSCLE MODEL}

The transformation from EMG to muscle activation is an essential process in classical approach. Especially in Hilltype models, it is dominant process because the estimated muscle force is assumed to be proportional to the muscle activation. How to obtain the activation level from EMG influences a lot in this approach. Here, we describe typical steps to perform this transform. One should keep in mind that most researchers use a subset of the described method.

\section{A. EMG Processing}

Here, we summarize the employed EMG processing method. For the detail, you can refer to the literature [8][11].

1) high-pass filtering of the raw EMG using zero-lag 4th Butterworth filter $(30 \mathrm{~Hz})$ to remove movement artifact

2) wave rectification

3) low-pass filtering with a $2-10 \mathrm{~Hz}$ cut-off frequency

4) normalization with the peak of Maximum Voluntary Contraction (MVC)

The normalized, rectified, filtered EMG is referred as $e(t)$. There exists a time delay for the muscle activation. The process of transforming $e(t)$ to neural activation $p(t)$, is called activation dynamics. When a muscle fiber is activated by a single Action Potential (AP), the muscle generates a twitch response. This response can be well represented by a critically damped linear second-order differential system. Its recursive discrete filter can be obtained as in Eq. 1 to calculate $p(t)$.

$$
p(t)=\gamma e(t-d)-\beta_{1} p(t-1)-\beta_{2} p(t-2)
$$

where $\mathrm{d}$ is the electromechanical delay and $\gamma, \beta_{1}$ and $\beta_{2}$ are the coefficients that define the second-order dynamics. To realize a positive stable solution, a set of constraints was employed, i.e. $\beta_{1}=C_{1}+C_{2}, \beta_{2}=C_{1} C_{2}$ where $\left|C_{1}\right|<$ $1,\left|C_{2}\right|<1$. In addition, the unit gain of this filter should be maintained by ensuring $\gamma-\beta_{1}-\beta_{2}=1$.

\section{B. Nonlinearization for neural to muscle activation}

Many researchers assume that the above $p(t)$ is a reasonable approximation of muscle activation. However, nonlinear relationship has been reported between individual muscle EMG and joint moment for some muscles especially at lower forces [18]. In studies on single motor units, multiple APs cause multiple twitch responses. If the time between APs decreases, the twitches start to merge into each other and the muscle force increases steadily. However, at high frequency the twitches get closer to tetanus, where no further force is produced even if the frequency increases. This means there is a nonlinear relationship between frequency and force for single motor units.

Therefore, nonlinearization from neural activation $p(t)$ to muscle activation $a(t)$ should be considered. As the simple and adequate solution, Lloyd and Besier [8] proposed following formulation:

$$
a(t)=\frac{e^{A p(t)}-1}{e^{A}-1}
$$

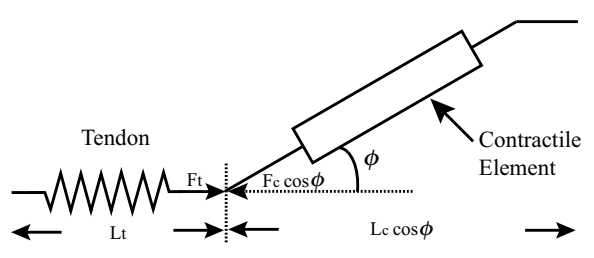

Fig. 1. typical muscle-tendon macroscopic model.

where $\mathrm{A}$ is the nonlinear shape factor allowed to vary between -3 and 0 , with $A=-3$ being highly exponential and $A=0$ being linear.

\section{Hill-type muscle model}

The muscle-tendon unit is modeled as a contractile element in series with a elastic tendon as in Fig. 1. The Hill-type muscle model is used to estimate the force $F_{c}(t)$ that can be generated by the contractile element with general form of the function by

$$
F_{c}(t)=a(t) f_{l}\left(\varepsilon_{c}\right) f_{v}\left(\dot{\varepsilon}_{c}\right) F_{0}^{m}
$$

where $\varepsilon_{c}$ is the strain of contractile element, $f_{l}\left(\varepsilon_{c}\right)$ and $f_{v}\left(\dot{\varepsilon}_{c}\right)$ are the normalized force-length and force-velocity relationship respectively. $F_{0}^{m}$ is the maximum isometric muscle force. $\phi$ is the pennation angle between the tendon and the muscle fibers. In this paper, only concentric contraction is considered, thus parallel elastic element is not introduced.

The force length relationship shows a Gaussian distribution around the optimal length and is formulated as

$$
f_{l}\left(\varepsilon_{c}\right)=\exp \left\{-\left(\frac{\varepsilon_{c}}{b}\right)^{2}\right\}
$$

where $b$ is a constant parameter.

$f_{v}\left(\dot{\varepsilon}_{c}\right)$ represents the relationship between velocity and normalized force. The muscle can contract at its maximum velocity $v_{\max }$ without load and slows down as the load increases. In the case of concentric contraction, this relationship is formulated as follows:

$$
f_{v}\left(\dot{\varepsilon}_{c}\right)=\frac{V_{s h}\left(v_{\max }+L_{c 0} \dot{\varepsilon}_{c}\right)}{V_{s h} v_{\max }-L_{c 0} \dot{\varepsilon}_{c}}
$$

where $V_{s h}$ is a constant parameter.

In every time step, fiber velocity should be solved and muscle fiber length can be calculated by forward integration using Runge-Kutta algorithm. Since the value for $\varepsilon_{c}$ has changed, the calculation should continue iteratively until the end of input time series of $a(t)$.

Muscle tendon parameters were adopted from Delp [19]. The optimal lengths of contractile element $\left(L_{c 0}\right)$ are $3 \mathrm{~cm}$ for soleus and $5.1 \mathrm{~cm}$ for gastrocnemius. The tendon slack lengths $\left(L_{t 0}\right)$ are $26.8 \mathrm{~cm}$ for soleus and $40 \mathrm{~cm}$ for gastrocnemius. For gastrocnemius, parameters are averages of two heads (med/lat). Only EMG of medial head is measured for its input, but the force of both heads is computed using the sum of maximum force of two heads as $F_{0}^{m}$. Also for $F_{0}^{m}$ and pennation angle $\phi$, these parameters are obtained from this reference, but final result is normalized by maximum contraction, so the effect of these parameters can be neglected. 


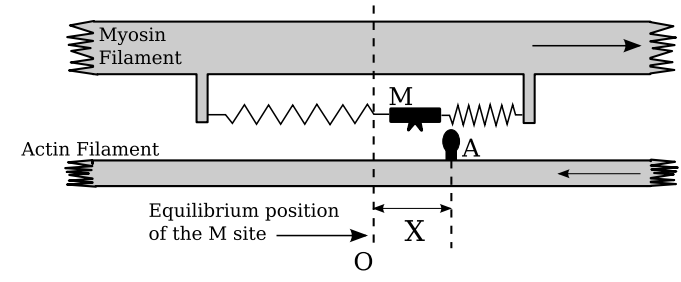

Fig. 2. Huxley Sliding filaments model.

\section{PHYSIOLOGICAL MUSCLE MODEL}

\section{A. Sarcomere scale}

All the sarcomeres are assumed to be identical, and the deformation of both sarcomere and muscle scale is proportional. If $S$ is the sarcomere length, we can write $\left(S-S_{0}\right) / S_{0}=\left(L_{c}-L_{c 0}\right) / L_{c 0}=\varepsilon_{c}$.

Huxley proposed that a cross-bridge between actine filaments and myosine heads could exist in two biochemical states, attached and detached states. He postulated that one myosine head could attach to only one actine site. Then, the dynamics of the fraction $n(y, t)$ of the attached cross bridges is given by

$$
\frac{\partial n}{\partial t}+\frac{S_{0}}{h} \dot{\varepsilon}_{c} \frac{\partial n}{\partial y}=f(y, t)[1-n(y, t)]-g(y, t) n(y, t)
$$

where $h$ is the maximum elongation of the myosine spring, $x$ the distance and $y$ the normalized distance between actine binding site and myosine head: $y=\frac{x}{h} \cdot n(y, t)$ is a distribution function representing the fraction of attached cross bridges relative to the normalized position $y$. To ensure a one way displacement, this attachment is considered possible only when $y$ is between 0 and $1 . S_{0} \dot{\varepsilon}_{C}$ represents the velocity of the actine filament relative to the myosine filament. $f$ and $g$ denote the rate functions of attachment and detachment respectively.

From the Huxley paper in 1957, this dynamics has never been drastically changed; researches focused mainly on the definition of $f$ and $g$. Several $f$ and $g$ functions have been defined and recently the chemical input was introduced by Bestel-Sorine [16] to modify the ability of the cross bridge to attach or not. Moreover, they proposed that these rates depend on the relative velocity between actine and myosine. Indeed, the higher the velocity is, the greater the probability to break bridges is. $f$ and $g$ can thus be defined by:

Contraction phase

$$
\left\{\begin{array}{l}
f(y, t)=U_{c} \\
g(y, t)=U_{c}+\left|\dot{\varepsilon}_{c}\right|-f(y, t)
\end{array}\right.
$$

\section{Relaxation phase}

$$
\left\{\begin{array}{l}
f(y, t)=0 \\
g(y, t)=U_{r}+\left|\dot{\varepsilon}_{c}\right|
\end{array}\right.
$$

$U_{c}$ and $U_{r}$ are the level of chemical kinetics under contraction and relaxation phase respectively. That can be resumed as below

$$
\begin{aligned}
& u(t)=\Pi_{c}(t) U_{c}+\left(1-\Pi_{c}(t)\right) U_{r} \\
& \Pi_{c}(t)=1 \text { during contraction, } 0 \text { else } \\
& (f+g)(y, t)=u(t)+\left|\dot{\varepsilon}_{c}\right|
\end{aligned}
$$

To complete the description at the sarcomere scale, we assume that the force generated by one attached cross bridge is modeled by a linear spring with a constant stiffness. All the cross bridge are in parallel so that the global stiffness and force generated by the whole sarcomere is proportional to the number of formed bridges. Let's note $k_{0}\left(\mathrm{Nm}^{-1}\right)$ the maximum stiffness obtained when all the available bridges are attached. Let's define $\xi(y, t)$ the elongation of a cross bridge due to the contribution of the global extension of the sarcomere, considering $\varepsilon_{c}(0)$ the initial value, and to the local distribution of elongations $y$ :

$$
\xi(y, t)=y+\frac{S_{0}}{h}\left(\varepsilon_{c}(t)-\varepsilon_{c}(0)\right)
$$

Stiffness and force generated by a muscle sarcomere is obtained by computing the first and second moment of the distribution $n(\xi(y, t), t)$. The moments are defined as:

$$
\begin{gathered}
k_{s}(t)=k_{0} \int_{-\infty}^{+\infty} n(\xi(y, t), t) d y \\
F_{s}(t)=k_{0} h \int_{-\infty}^{+\infty} \xi(y, t) n(\xi(y, t), t) d y
\end{gathered}
$$

From Eq.6, it can be rewritten as follows:

$$
\begin{aligned}
& \dot{k}_{s}=-(f+g)(y, t) k_{s}+k_{0} f(y, t) \\
& \dot{F}_{s}=-(f+g)(y, t) F_{s}+k_{s} S_{0} \dot{\varepsilon}_{c}+\frac{1}{2} k_{0} h f(y, t)
\end{aligned}
$$

\section{B. Myofiber and muscle scale}

This set of differential equations is easily extended to the whole muscle fiber considering that each fiber is composed of identical sarcormeres in series. Then $k_{f}=k_{s} \frac{S_{0}}{L_{c 0}}$ and $F_{f}=$ $F_{s}$. In addition, the maximum available cross bridges could be varied depending on the relative length of the contractile element. It is known as the force-length relationship $\left(f_{l}\left(\varepsilon_{c}\right)\right)$. Contrary to previous studies where this effect is introduced at the macroscopic level, we take into account this relation at the microscopic scale. Indeed, this relation is directly linked to the maximum available actine and myosine sites [14]. For the stiffness $k_{f}$ and the force $F_{f}$ at the fiber scale we get including Eq.9:

$$
\begin{aligned}
& \dot{k}_{f}=-\left(u+\left|\dot{\varepsilon}_{c}\right|\right) k_{f}+\frac{S_{0}}{L_{c 0}} k_{0} \Pi_{c}(t) U_{c} f_{l}\left(\varepsilon_{c}\right) \\
& \dot{F}_{f}=-\left(u+\left|\dot{\varepsilon}_{c}\right|\right) F_{f}+k_{f} L_{c 0} \dot{\varepsilon}_{c}+\frac{1}{2} k_{0} h \Pi_{c}(t) U_{c} f_{l}\left(\varepsilon_{c}\right)
\end{aligned}
$$

Next, the recruitment process is introduced to muscle scale because MUs have an all-or-nothing response to APs. At each contraction phase, the recruitment ratio is updated but remains constant during its phase itself. Let's define $k_{c}$ and $F_{c}$ the stiffness and the force for whole contractile element, and $N$ the number of all MUs. The recruited number is written as $\alpha N$ using recruitment ratio $\alpha$. Finally the desired model of contractile element is a set of differential equations as below:

$$
\begin{aligned}
& \dot{k}_{c}=-\left(u+\left|\dot{\varepsilon}_{c}\right|\right) k_{c}+\alpha k_{m} \Pi_{c}(t) U_{c} \\
& \dot{F}_{c}=-\left(u+\left|\dot{\varepsilon}_{c}\right|\right) F_{c}+\alpha F_{m} \Pi_{c}(t) U_{c}+k_{c} L_{c 0} \dot{\varepsilon}_{c}
\end{aligned}
$$

where $k_{m}=S_{0} N k_{0} f_{l}\left(\varepsilon_{c}\right) / L_{c 0}, F_{m}=N k_{0} h f_{l}\left(\varepsilon_{c}\right) / 2$.

For the macroscopic representation, the same configuration with Fig. 1 is used including the muscle tendon parameters. The contractile element is only replaced with the above nonlinear differential equations. 


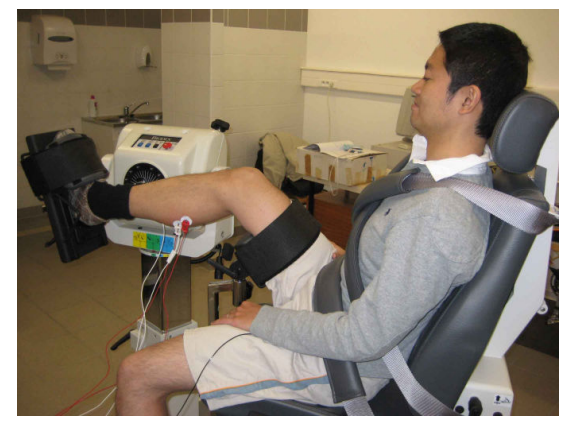

Fig. 3. Appearance of the experiment to measure EMGs and forces.

\section{RESULTS}

Two subjects were seated on a chair with their right foot fixed on a Biodex dynamometer (Biodex Medical Systems, Inc., New York, USA) as shown in Fig.3. The torque around ankle joint was measured when it is voluntarily generated for the extension position. For EMG measurements, bipolar surface $\mathrm{Ag} / \mathrm{AgCl}$-electrodes were placed on the muscle belly of the medial Gastrocnemius (GAS) and Soleus (SOL). Synchronous acquisition of the force and differential EMG signal was done with a sample frequency of $2048 \mathrm{~Hz}$ by the Biopac MP100 (Biopac Systems, Inc., Santa Barbara, USA).

Here, isometric moment was estimated only from EMG signals by the classical Hill approach and the proposed physiological based muscle model. The predicted torque was compared with the directly measured torque around the ankle by Biodex system. For this preliminary trial, we make the comparison for the normalized torque against the one of maximum voluntary contraction (MVC). For the conversion from force to moment, moment arm for each muscle has to be multiplied by the muscle force. The normalized moment was used in this result, therefore the absolute value of moment arm can be omitted. However, we still need to know the ratio of the contribution among both muscles of GAS and SOL for the resultant moment. The moment arm was estimated from the Hawkins data [20] from the joint angle in the measured condition. The contribution ratio was calculated using the literature values of Delp [19] like maximum force by moment arm considering pennation angle. The resultant ratio is $\mathrm{MG}$ 0.41 vs SOL 0.59 . The SUM in the shown graphs is plotted using the ratio as the sum of two muscles.

For the classical approach, the obtained EMG data was processed as explained in Section II. The muscle force was calculated considering force-length and force-velocity relationship as in Eq.3. Finally, normalized estimated torque by classical Hill approach was obtained as shown in Fig. 4. The normalized data of measured torque (red), normalized estimated torque of SOL (green), normalized estimated torque of GAS (magenta) and the sum of two muscles (blue) are plotted.

For the new model, the rectified EMG was low-pass filtered with $30 \mathrm{~Hz}$ cut-off frequency. Then, chemical input $u(t)$ was created by thresholding the extracted EMG signals as shown in Fig. 5. The thresholding can be assumed as muscle cell's all-or-nothing response to Action Potential (AP). Muscle contraction is initiated by an AP along the

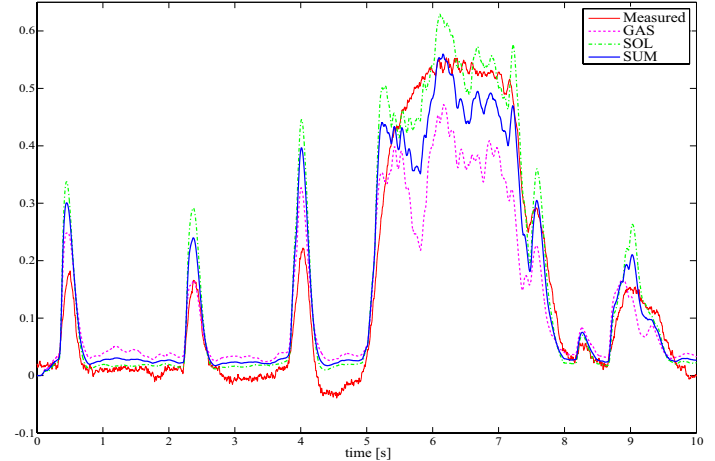

Fig. 4. Normalized estimated torques by classical Hill approach and measured torque (red:measured, magenta:GAS, green:SOL, blue:SUM).
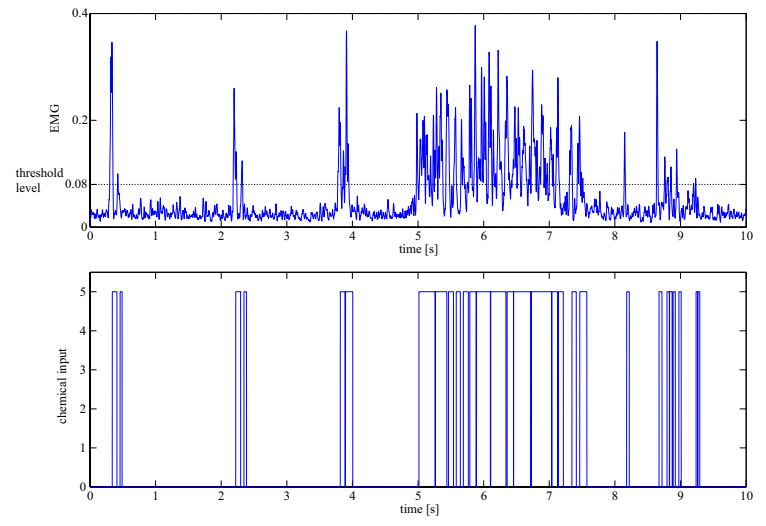

Fig. 5. Generation of chemical input. Top: filtered rectified EMG signal, bottom: generated chemical input by thresholding for GAS.

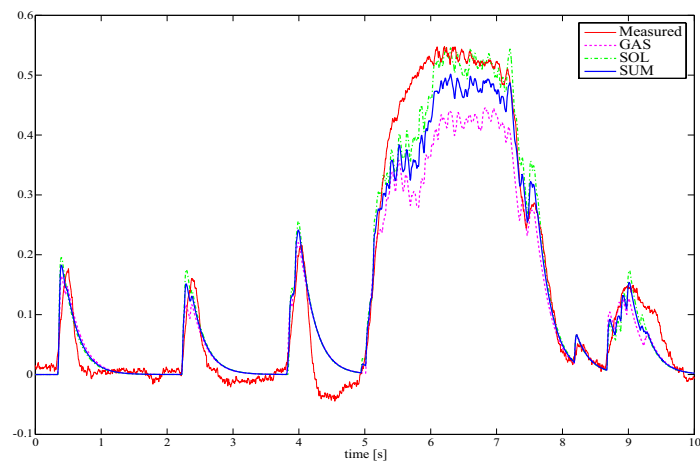

Fig. 6. Normalized estimated torque by physiological muscle model and measured torque (red:measured, magenta:GAS, green:SOL, blue:SUM).

muscle fiber membrane, which goes deeply into the cell through T-tubules. It causes calcium releases that induce the contraction process when the concentration goes above a threshold and is sustained till the concentration goes down this threshold again. We use a delayed model to take into account the propagation time of the AP and an average delay due to the calcium dynamics. It is corresponding to $e(t)$ to $p(t)$ conversion in Hill-type approach. The same value of time delay was used for both estimation processes. For recruitment ratio $\alpha$, the amplitude of normalized EMG is used.

The generated input command $u(t)$ was given to the contractile element of physiological model and the active stiffness $k_{c}$ and the muscle force $F_{c}$ were computed. Finally, normalized estimated torque by physiological muscle model 


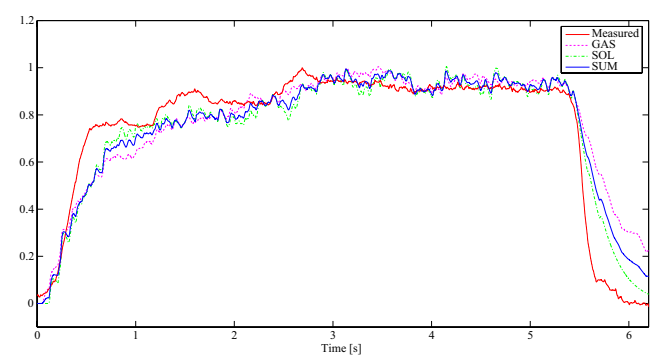

Fig. 7. Normalized torques for MVC by physiological muscle model.

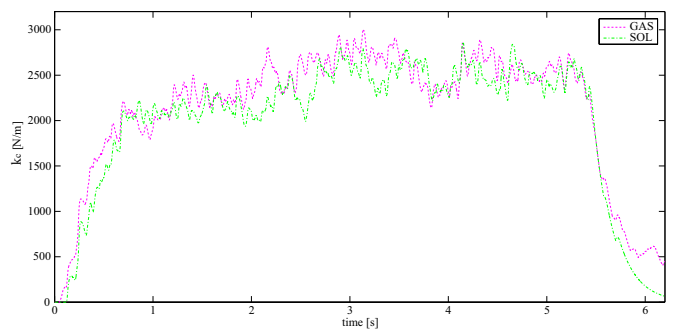

Fig. 8. Estimated active stiffness for MVC by physiological muscle model.

could be obtained as shown in Fig. 6. In order to confirm the estimation ability both for short-term contraction and longterm contraction, the result which includes two type contractions is presented here. The obtained RMS errors are given in Table I for both contraction types and by both estimation approaches. The estimation result by physiological model for MVC can be seen as in Fig. 7 and 8. Same thresholding level as in Fig. 5 is used. In the physiological model, active stiffness also can be estimated along with the muscle force.

TABLE I

RMS ERRORS BETWEEN THE MEASURED AND THE ESTIMATED

\begin{tabular}{cccccc}
\hline \multirow{2}{*}{ Subject } & \multicolumn{2}{c}{ Modified Hill } & & \multicolumn{2}{c}{ Full Physiology } \\
\cline { 2 - 3 } \cline { 5 - 6 } & short term & long term & & short term & long term \\
\hline \hline 1 & 0.0519 & 0.0523 & & 0.0313 & 0.0420 \\
2 & 0.1015 & 0.0823 & & 0.0705 & 0.0557 \\
\hline
\end{tabular}

\section{DISCUSSION}

The first results highlight the effectiveness of EMG-toforce estimation with physiological based muscle model and its feasibility. In this paper, the identification of internal parameters is not executed. For the classical approach, it is known that the optimization to fit the experimental data is possible using the calibration technique as written in [8]. Theoretically, it can be said that the optimization can be done also for the proposed physiological model. Using the force response of rabbit skeletal muscle by electrical stimulation, identification of the physiological model could be achived in [21]. Once the optimization is carried out, the comparison in absolute force scale would be available.

Here, we estimated the normalized torque by both classical Hill-Zajac model and novel physiological muscle model based on the same EMG signals in voluntary case. For the common parameters between two approaches, the same value was used for its calculation like the value of electromechanical delay $40 \mathrm{~ms}$ in calcium dynamics and constant value in force-length relationship. In order to keep the similar condition, the common macroscopic model as in Fig. 1 was used for both approaches. Only the contractile element was replaced. Thus, the dynamics for force generation from EMG signal is different as presented in Section II and III.

The aim to introduce the new physiologically detailed model is not for the higher precision of force estimation. Hill-type muscle is a phenomenological model based on the experimental fact to represent the resultant relation between length, velocity and force. There is no link to the microscopic physiology. Even if the estimation ability is approximately equivalent, it is meaningful to understand and capture the muscle dynamics with more detailed representation. In this paper, it is still qualitative validation, but same kind of force generation could be obtained with the newly proposed physiological model against voluntary EMG signal. If we take a look carefully at the result of classical model as in Fig. 4 , you can find the larger error for the estimation of shortterm contraction. The reason of this error can be considered as follows. It is known that there is a nonlinear relationship between frequency in contraction and force for single motor units. In classical approach, this frequency dependency is tried to be offset only by the nonlinear conversion from $p(t)$ to $a(t)$ as written in Section II.B. This nonlinearization was proposed recently to modify Hill model. Originally this process has not been introduced in the initial method of Hilltype approach. However, even with the modification, it is not a time function so that it still can not correspond to the varied frequency of muscle contraction. In the proposed approach, the frequency property is internally integrated in the generation process of chemical input $u(t)$.

Additionally, the signal measured in EMG is the summation of the signals of all different motor units (MU). So even in the short-term tetanic contraction, the amplitude of EMG is the same level with the amplitude in longterm tetanic contraction. However, the resultant force of short-term contraction is actually much less than the one of long-term contraction. It means that there is somewhat time hysteresis regarding the neural command. In classical approach, neural activation $p(t)$ is dominantly decided by the low-pass filtering with $2-10 \mathrm{~Hz}$ cut-off frequency. The choice of the cut-off frequency is very sensitive to the obtained $p(t)$. Then, $p(t)$ is more or less proportional to the resultant force in Hill-type model. It does not include an effect of time hysteresis, therefore classical approach can not estimate well both the short-term and long-term contraction in the same time with a certain cut-off frequency for EMG processing. In the proposed approach, the derivative of the contraction force is directly given by the neural command and it brings time hysteresis in force generation. The choice of threshold level is not so sensitive to the input generation. It is interesting to see this kind of effect along with the introduction of Huxley cross-bridge representation.

Furthermore, the physiological model verifies the well established properties observed for the muscle's behavior: i) the force-length relation is included in the definition of Eq.13, ii) the force-velocity relation can be expressed in isotonic and tetanic conditions. Before the isotonic contraction 
could occur, the muscle contracts in an isometric condition until the force generated by the muscle equilibrates the imposed one. Then, isotonic contraction becomes possible. When we assume $\dot{F}_{c}=0$ in Eq.14, it can be rewritten as $F_{c}=\left(\alpha F_{m} U_{c}+k_{c} L_{c 0} \dot{\varepsilon}_{c}\right) /\left(U_{c}+\left|\dot{\varepsilon}_{c}\right|\right)$.

At the beginning of this phase $(\mathrm{t}=0), k_{c}(0) \neq 0$ but depends on the initial isometric contraction phase. If we define $A(0)=\frac{L_{c 0} k_{c}(0)}{U_{c} \alpha F_{m}}, B=\frac{1}{U_{c}}$, in a concentric contraction where $\dot{\varepsilon}_{c}<0$, we get a Hill type force-velocity relation:

$$
F_{c}=\alpha F_{m} \frac{1+A(0) \dot{\varepsilon}_{c}}{1-B \dot{\varepsilon}_{c}}
$$

You can find the correspondence in the relation of Eq. 5. It can be verified that this physiological muscle model integrates the force-velocity relation naturally from the consideration of actin-myosin cross bridge.

Finally, EMG signal of one muscle is formed of the contributions of all different Motor Units (MUs) active at a certain time. During voluntary contraction, in general the active MUs will have different fire frequencies and their activity is not synchronized. Therefore, in strict sense chemical input $u(t)$ of the proposed model also should not be synchronized. However in this first trial, one chemical input was used for the calculation to confirm the validity of one model as the single MU. The resultant force in this work can be seen as the contribution of representative MUs. In reality, the muscle is consisted of numerous MUs. If we consider the extracted EMG signal as mean MU action potential and the unsynchronized chemical input $u(t)$ are generated for each MU with the time delay of Gaussian distribution, the more realistic modeling would be possible. For the future work, we will try the simulation with many sets of model corresponding to multiple MUs for one muscle.

\section{CONCLUSION}

In this paper we have presented a method that allows to estimate the muscle force from EMG signal with physiology based model which has the link to underlying microscopic filament dynamics. The proposed method features:

- a novel physiologically detailed model for EMG-toforce estimation instead of a phenomenological Hilltype muscle model,

- the estimation improvement both for short-term and long-term contraction with the integration of the frequency property of neural activation and muscle force,

- the consideration of firing rates of motor units in the generation of chemical command input.

The summation of electrical activity created by each MU appears in EMG. Strictly, EMG signals should be decomposed into constituent MU action potentials to obtain the map of MU firings. However, the generation of chemical command by thresholding EMG can at least capture the fact that the firing rates of MUs increase, the twitches associated with each firing will eventually fuse to yield large force. Future work will focus on increasing the number of tests and the further interpretation of neuromuscular system both in voluntary and artificial activation in FES. Identification of internal parameters would also contribute to the optimized modeling for different properties of subjects.

\section{ACKNOWLEDGMENTS}

We would like to thank A. Varray and M. Papaiordanidou at Univ. of Montpellier I for their help at the experiments.

\section{REFERENCES}

[1] T. Flash, N. Hogan. "The coordination of arm movements: an experimentally confirmed mathematical model”. Journal of Neuroscience, 5(7):1688-1703, 1985.

[2] M. Kawato, Y. Maeda, Y. Uno and R. Suzuki. "Trajectory formation of arm movement by cascade neural network model based on minimum torque-change criterion". Biological Cybernetics, 62(4):275-288, 1990.

[3] S. Smaga. "Tremor". American Family Physician, 68, 1545-1552, 2003.

[4] R. Kobetic, R.J. Triolo, J.P. Uhlir, C. Bieri, M. Wibowo, G. Polando, E.B. Marsolais, J.A. Davis, Implanted Functional Electrical Stimulation System for Mobility in Paraplegia:A Follow-Up Case Report, IEEE Trans. on Rehabilitation Eng., vol. 7, no 4, pp 390-398, 1999.

[5] D. Guiraud, T. Stieglitz, K.P. Koch, J.L. Divoux, P. Rabischong, "An implantable neuroprosthesis for standing and walking in paraplegia: 5-year patient follow-up", J. Neural Eng., vol. 3, pp 268-275, 2006.

[6] S.L. Delp, F.C. Anderson et al, "OpenSim: Open-Source Software to Create and Analyze Dynamic Simulations of Movement", IEEE Trans. on Biomedical Engineering, vol. 54, no. 11, pp 1940-1950, 2007.

[7] Y. Nakamura, K. Yamane, Y. Fujita, and I. Suzuki, ”Somatosensory Computation for Man-Machine Interface From Motion-Capture Data and Musculoskeletal Human Model", IEEE Trans. on Robotics, vol. 21, no. 1, pp 58-66, 2005.

[8] D.G. Lloyd and T.F. Bessier, "An emg-driven musculoskeletal model to estimate muscle forces and knee joint moment in vivo", Journal of Biomechanics, no. 36, pp. 765-776, 2003.

[9] A.V. Hill, "The heat of shortening and the dynamic constants in muscle", Proceeding of the royal society, London, Sre. B, vol. 126, pp. 136-195, 1938.

[10] F.E. Zajac "Muscle and tendon: properties, models, scaling and application to biomechanics and motor control". CRC Critic. Rev. in Biomed. Eng. 17: 359-411, 1989.

[11] T.S. Buchanan, D.G. Lloyd, K. Manal and T.F. Bessier, "Neuromusculoskeletal modeling: estimation of muscle forces and joint moments and movements from measurements of neural command", Journal of Applied Biomechanics, no. 20, pp. 367-395, 2004.

[12] G. Venture, K. Yamane, and Y. Nakamura, "In-vivo estimation of the human elbow joint dynamics during passive movements based on the musculo-skeletal kinematics computation", IEEE/Int. Conf. on Robotics and Automation, Orlando, USA, pp. 2960-2965, 2006.

[13] A.F. Huxley, Muscle structure and theories of contraction. Progress in Biophysics and Biophysical Chemistry, vol. 7, pp. 255-318, 1957.

[14] J.L. Leeuwen, Optimum Power Output and Structural Design of Sarcomeres, J. of Theor. Biol. 19, 229-256, 1991.

[15] G.I. Zahalak, A distribution-moment approximation for kinetic theories of muscular contraction. Mathematical Biosciences, vol. 55, pp. 89-114, 1981.

[16] J. Bestel, M. Sorine, A differential model of muscle contraction and applications. In schloessmann Seminar on Mathematical Models in Biology, Chemistry and Physics, Max Plank Society, Bad Lausick, Germany, May 19-23, 2000.

[17] H. El Makssoud, D. Guiraud, P. Poignet, Mathematical muscle model for Functional Electrical Stimulation control strategies. Proc. IEEE/Int. Conf. on Robotics and Automation, pp. 1282-1287, 2004.

[18] K. Manal, T.S. Buchanan, "A one-parameter neural activation to muscle activation model", Journal of Biomechanics, vol. 36, pp. 11971202, 2003.

[19] S.L. Delp, "Surgery simulation: a computer graphics system to analyze and design musculoskeletal reconstructions of the lower limb", Dissertation, Stanford University, CA, USA, 1990.

[20] D. Hawkins and M. Hull, "A method for determining lower extremity muscle-tendon lengths during flexion/extension movements", Journal of Biomechanics, vol. 23, pp. 487-494, 1990.

[21] M. Hayashibe, P. Poignet, D. Guiraud, H. Makksoud, Nonlinear Identification of skeletal muscle dynamics with Sigma-Point Kalman Filter for model-based FES. Proc. IEEE/Int. Conf. on Robotics and Automation, pp. 2049-2054, 2008. 